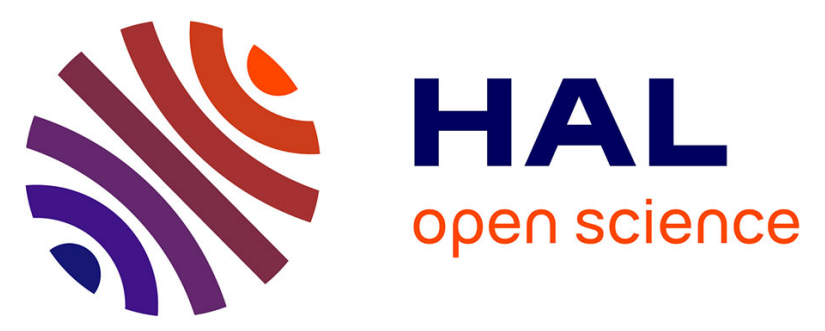

\title{
Théorie du comportement adaptatif et agriculture familiale
}

\author{
E. Chia, M. Petit, J. Brossier
}

\section{To cite this version:}

E. Chia, M. Petit, J. Brossier. Théorie du comportement adaptatif et agriculture familiale. Gasselin P. (ed.), Choisis J.P. (ed.), Petit S. (ed.), Purseigle F. (ed.), Zasser-Bedoya S. (ed.). L'agriculture en famille: travailler, réinventer, transmettre, EDP Sciences, pp.81-100, 2014, 978-2-7598-1192-2 978-27598-1765-8. 10.1051/978-2-7598-1192-2.c006 . hal-02163636

\section{HAL Id: hal-02163636 https://hal.science/hal-02163636}

Submitted on 5 Jun 2020

HAL is a multi-disciplinary open access archive for the deposit and dissemination of scientific research documents, whether they are published or not. The documents may come from teaching and research institutions in France or abroad, or from public or private research centers.
L'archive ouverte pluridisciplinaire HAL, est destinée au dépôt et à la diffusion de documents scientifiques de niveau recherche, publiés ou non, émanant des établissements d'enseignement et de recherche français ou étrangers, des laboratoires publics ou privés. 


\title{
Théorie du comportement adaptatif et agriculture familiale
}

\author{
Eduardo Chia ${ }^{1}$, Michel Petit ${ }^{2}$, Jacques Brossier ${ }^{3}$
}

\section{Introduction ${ }^{4}$}

La diversité des unités de production agricole dans le monde est particulièrement frappante aujourd'hui. Elle est d'ailleurs remarquée par de nombreux auteurs. À cette diversité dans l'espace, il faut ajouter la profonde transformation des unités de production dans de nombreux pays, où l'évolution de l'agriculture a été très rapide et porteuse de multiples débats. En cinquante ans, on est passé dans les discours, en France notamment d'une agriculture dominée par les "paysans ", puis par les " exploitants familiaux ", à une agriculture d' " entrepreneurs agricoles ». Ailleurs, on parle aussi bien de petite agriculture, d'entreprises agricoles, d'entreprenariat agricole, d'agrobusiness...

Ainsi, l'agriculture familiale, célébrée en 2014 - déclarée année mondiale de l'agriculture familiale par l'ONU - n'est plus la seule, au niveau international, à occuper les espaces, à produire des biens et services agricoles, à générer des emplois, à participer à la dynamique territoriale. Il est d'ailleurs probable que les formes familiales n'ont jamais eu un monopole en la matière. Pourtant dans de très nombreuses situations, les liens entre famille et unité de production continuent de jouer un rôle important, souvent même crucial. Et ces liens justifient bien de continuer de parler d'agriculture familiale, même si les formes de celle-ci continuent de se diversifier. À côté des formes individuelles, on assiste aussi à l'émergence de formes collectives, comme les GAEC, les EARL, les CUMA ${ }^{5}$ en France, et beaucoup d'autres ailleurs, telle que par exemple la "capitalización " en Argentine, une forme certes moins

1. INRA, UMR Innovation, 34060-Montpellier, France, chia@supagro.inra.fr

2. Professeur, Institut agronomique méditerranéen, 34093 Montpellier, France, petit@iamm.fr

3. Retraité, ancien président du centre Inra de Dijon et ancien directeur de recherche de l'équipe de recherche de l'INRA SAD, 21000 Dijon, France, brossier48@gmail.com

4. Nous dédions ce chapitre à Éric Marshall, compagnon au long cours, décédé en 2012.

5. GAEC : Groupement agricole d'exploitation en commun; EARL : Exploitation agricole à responsabilité limitée ; CUMA : Coopérative d'utilisation de matériel agricole.

Ce chapitre de l'ouvrage L'agriculture en famille : travailler, réinventer, transmettre est publié en Open Access sous licence creative commons CC-BY-NC-ND permettant l'utilisation non commerciale, la distribution, la reproduction du texte, sur n'importe quel support, à condition de citer la source. 
institutionnalisée d'action collective mais néanmoins réelle. En Afrique soudanosahélienne aussi, les groupements de producteurs familiaux se multiplient tout en prenant des formes juridiques variées. De nombreux auteurs ont témoigné ou rendu compte de cette prolifération de nouvelles formes d'unités de production agricole dans de nombreuses régions du monde.

Ainsi la typologie proposée récemment par B. Hervieu et F. Purseigle (2013) pour rendre compte de cette diversité nous a paru illustrative. Ces auteurs proposent trois grandes catégories : agricultures familiales, agricultures de firme et agricultures de subsistance. Ces catégories sont, elles-mêmes, décomposables en sous-catégories. Nous avons élaboré un tableau basé sur cette catégorisation et résumant les principaux apports de ces auteurs (Tab. 1). Il présente de manière synthétique la diversité des unités de production agricole et illustre ainsi la diversité des situations agricoles tout en étant basé sur des critères de classification qui sont simples, essentiellement des critères de structure (surface, nature de la main-d'œuvre et sources de revenus). Toutefois, rien n'est dit sur les processus de décision au sein des unités de production, même si Hervieu et Purseigle parlent de dix " façons de travailler la terre ", ce qui peut implicitement renvoyer aux modèles de décision. Nous reviendrons ci-dessous sur ce point, capital pour nous.

Il est clair que les exploitations agricoles, plus ou moins familiales, sont encore très nombreuses dans le monde (un exemple parmi d'autres, on en compte 17 millions en Amérique latine (Sotomayor et al., 2011). De nombreux travaux ont montré que cette agriculture familiale est multifonctionnelle assurant plusieurs rôles importants. Outre qu'elle assure une part importante de la production, elle a une fonction environnementale (en occupant des espaces souvent difficiles à aménager, contribuant à la lutte contre la désertification, les incendies, valorisant le territoire avec le tourisme ${ }^{6} \ldots$ ), elle garantit la sécurité alimentaire et joue un rôle social évident sans oublier son rôle culturel aujourd'hui bien reconnu. Ainsi des chercheurs et des hommes politiques mettent en avant les capacités d'adaptation et de flexibilité des unités de production familiales ou d'exploitations de petite taille pour s'adapter aux nouvelles conditions de production en particulier pour respecter les contraintes environnementales. En France, par exemple, la nouvelle loi agricole (2014) cherche à (re)donner une place importante aux exploitations familiales de taille moyennes en métropole et de petites tailles dans le départements d'Outre-mer (DOM) pour contribuer à la mise en place des nouveaux systèmes performants grâce à l'intensification écologique (Guillou, 2013). Il est en effet probable que la transition écologique ne pourra faire l'économie d'une réflexion sur le rôle de ces agricultures dans les nouveaux régimes de production agricole.

6. Par exemple, nous parlons dans les Vosges " d'entrepreneurs multifonctionnels " pour désigner une catégorie d'éleveurs très actifs et dynamiques, pour qui les activités liées au tourisme apportent une part importante de leurs revenus, et qui contribuent beaucoup à l'entretien des paysages (Brossier et al. 2008). 


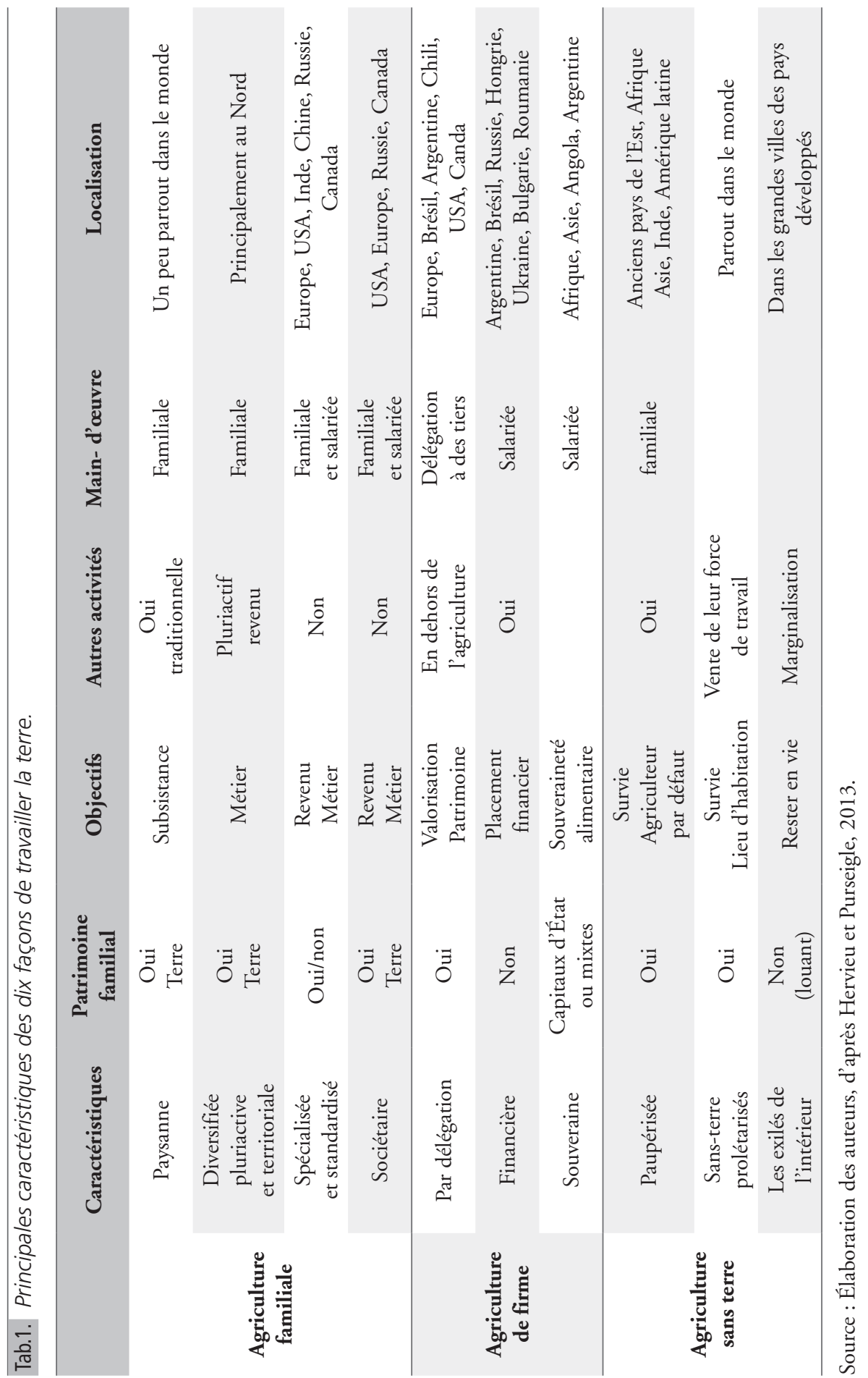


L'analyste est souvent désarmé face à cette diversité : comment l'appréhender ? Comment en rendre compte? Quant aux acteurs multiples, impliqués de façons variées dans des relations avec les agriculteurs, ils ont aussi besoin d'outils conceptuels pour comprendre l'objet de leurs actions et guider celles-ci. Pour cela, ils ont besoin de clés pour comprendre comment fonctionnent ces exploitations si diverses. Le point de vue que nous souhaitons défendre dans ce chapitre est que la théorie du comportement adaptatif des agriculteurs (TCA), que nous avons élaborée et proposée il y a près de quarante ans, reste dans la perspective qui vient d'être évoquée un outil analytique encore très utile aujourd'hui. Focalisée sur les décisions des agriculteurs (comment elles se prennent? quels en sont les déterminants ?), cette théorie est d'application générale et permet de rendre compte des façons dont ces différents types d'exploitation fonctionnent. Elle s'applique à de nombreuses formes d'unités de production agricole, tout particulièrement les formes familiales mais pas exclusivement. Sa principale limite est d'ailleurs liée à ce caractère général : la TCA n’est pas vraiment appropriée pour rendre compte au mieux des transformations sociales affectant l'agriculture, même si elle peut y contribuer.

Pour étayer notre point de vue, nous rappellerons d'abord ce qu'est la TCA. Nous exposerons ensuite en quoi cette théorie a été une référence utile dans le passé pour la rénovation pédagogique de l'enseignement agricole en France dans les années 1980 et 1990, pour l'analyse concrète de divers débats sur le conseil agricole en France et à l'étranger et pour répondre à des problèmes spécifiques de politique publique. Ceci nous permettra enfin de proposer quelques pistes pour l'avenir.

\section{Qu'est-ce que la théorie du comportement adaptatif ?}

La TCA a été développée en référence à la théorie économique de la production, telle que formulée par exemple par Bradford et Johnson (1953) dans leur manuel. Dans cet ouvrage sur la gestion des exploitations agricoles, devenu un classique, les auteurs avaient jugé important d'inclure plusieurs chapitres théoriques sur ce sujet $^{7}$. De la même façon, quelques vingt années plus tard, c'est notre implication dans la gestion et surtout dans la formation à la gestion qui nous a amenés à la nécessité d'un approfondissement théorique. Nous exposerons dans un premier temps cette filiation avec la théorie économique de la production, y compris ses développements les plus récents dans les années 1970. Nous montrerons ensuite en quoi la TCA constitue un dépassement des principales limites de la théorie néoclassique de la production, à savoir le caractère exogène de la fonction de production et

7. Les mêmes chapitres, traduits par J.-C. Tirel, ont aussi été insérés dans le célèbre manuel de J. Chombart de Lauwe et al. (1963). Cependant, les travaux empiriques de G. Johnson et de ses collègues (Johnson et al., 1961) sur les pratiques de gestion des agriculteurs dans les années 1950 et 1960 ont beaucoup contribué à nous sensibiliser aux limites de cette théorie économique de la production en tant que fondement théorique de la gestion des exploitations agricoles. 
de la fonction d'objectif. Enfin, nous indiquerons quelques pistes d'ouverture vers d'autres disciplines que l'économie.

\section{Filiation avec la théorie économique de la production}

La théorie de la production, avec sa célèbre loi des rendements décroissants, est une des pierres angulaires de la théorie économique, même si cela n'est pas reconnu par tous les économistes. Elle a deux fonctions liées mais bien distinctes. La première est celle de fournir une base théorique à la gestion des unités de production, autrement dit les exploitations agricoles pour ce qui nous concerne. Mais pour la plupart des économistes, c'est la seconde qui a été privilégiée à savoir celle d'être une composante indispensable de toute théorie économique visant à analyser le fonctionnement de l'économie dans son ensemble. Ainsi, la théorie des marchés est fondée sur la confrontation de l'offre et de la demande, et la théorie de l'offre est directement fondée sur la loi des rendements décroissants. Même au niveau macroéconomique, où l'on met souvent l'accent sur les variations de la demande globale, celle-ci doit être confrontée à une offre globale pour expliquer le chômage et l'inflation. Rappelons que les différents concepts de productivité sont directement issus de la théorie économique de la production.

Résumée à l'essentiel, la théorie de la production répond à une série de questions (quoi produire ? Combien ? Comment ? Avec quelles ressources? En quelles quantités ? Avec quelles techniques ?) en focalisant l'attention sur les décisions des producteurs individuels, chacun d'eux étant confronté à ces questions pour lui-même. L'hypothèse fondamentale est que le producteur prend ces décisions de telle sorte que son profit soit maximum dans le cadre des contraintes auxquelles il est soumis quant aux ressources qu'il peut mobiliser et des lois techniques de la production. Très tôt, les économistes agricoles ont suggéré de substituer la maximisation du revenu agricole à celle du profit pour tenir compte de la spécificité des exploitations agricoles familiales. Dans ces exploitations, une part importante des facteurs de production (travail, terre et capital) est en quelque sorte fournie directement par la famille sans passer par des marchés. Dans ce cas, le profit n'a qu'un sens très abstrait et, surtout, la maximisation du revenu agricole et celle du profit sont formellement équivalentes.

Deux autres prolongements principaux de cette théorie ont été développés et intégrés dans les années 1960-1970, d'une part la prise en compte du risque et d'autre part ce que l'on a alors appelé la "nouvelle économie du ménage " (Nerlove, 1974). Suite à des tentatives diverses pour rendre compte de la façon dont les producteurs prennent en compte le risque dans leurs décisions, un consensus s'est dégagé parmi les économistes (Dillon, 1971) pour substituer à la maximisation du profit, celle d'une fonction d'utilité dépendant à la fois du profit et du risque. Cette fonction d'utilité rend compte de l'arbitrage que font les individus entre recherche du profit et recherche de sécurité, autrement dit, en termes prosaïques, pourquoi la plupart des gens sont prêts à payer une prime d'assurance pour réduire les risques qu'ils encourent. La " nouvelle » économie du ménage a commencé par une application 
de la théorie de la production au fonctionnement économique du ménage, qui n'était vu auparavant que comme l'unité de base de la théorie de la consommation. Son principal intérêt est de traiter le temps dont dispose tout un chacun comme une ressource productive faisant l'objet d'arbitrages entre usages possibles, pour ce qui nous concerne le travail dans l'exploitation agricole, les soins aux enfants, la formation, les loisirs, etc. Là encore, le modèle est élargi en enrichissant les arguments de la fonction d'utilité à maximiser dans le cadre de contraintes liées aux limites des ressources mobilisables, y compris le propre temps des membres du ménage. Ce développement théorique, qui met l'accent sur la croissance de la valeur économique du temps dont nous disposons, fonde en quelque sorte la théorie du capital humain développée par Schultz (1972). Il a permis une interprétation originale des déterminants économiques de nombreux changements dans nos modes de vie, y compris les changements dans les habitudes alimentaires, et même de la baisse des taux de fertilité humaine (Becker, 1965).

\section{La TCA comme dépassement des limites de la théorie économique de la production}

C'est comme base théorique de la gestion des exploitations agricoles que les limites de la théorie économique de la production sont les plus gênantes. Il s'agit d'une théorie statique, au sens donné à ce terme par les économistes par opposition aux théories dynamiques rendant compte des évolutions dans le temps. Par exemple, la théorie répond aux questions : " quoi produire ? Combien ? Comment ? etc. " en les traitant comme solutions simultanées d'un système d'équations. Or pour un agriculteur, les décisions à prendre pour conduire une exploitation s'échelonnent dans le temps en fonction du déroulement des travaux, des saisons etc. Il en résulte une tendance à négliger les décisions au jour le jour, dont on sait pourtant qu'elles sont cruciales dans le revenu d'un agriculteur. De fait, l'expérience a montré que la théorie statique de la production ne constitue pas une bonne base pour comprendre et analyser les décisions des agriculteurs, en particulier celles pour le long terme, comme le choix des investissements et les décisions en situation de risques mal connus comme l'adoption des innovations techniques.

La TCA a proposé de recourir à des concepts nouveaux, du moins en économie, pour dépasser ces limites : ceux de projet, de situation, de perception et d'adaptation. Il ne nous paraît pas nécessaire de présenter ici l'ensemble de la théorie TCA, que l'on trouvera dans plusieurs articles et ouvrages ${ }^{8}$. Nous nous limiterons ici à en présenter les grandes lignes (Fig. 1). Le point de départ est la reconnaissance du fait que, pour être mise en œuvre, toute décision d'action (par exemple le choix d'un assolement) requiert une série d'actions plus élémentaires (choisir la parcelle,

8. On trouvera une formulation assez complète dans l'ouvrage de référence (aussi un manuel d'enseignement) de Brossier et al. (1997, réédité en 2002). Il s’agit du résultat d'une élaboration collective sur plusieurs années, les principaux concepts ayant déjà été proposés dans plusieurs articles antérieurs dont on trouvera les références dans cet ouvrage. 
choisir la variété, implanter telle culture dans telle parcelle) requérant chacune une ou plusieurs décisions. À chaque décision correspond la poursuite d'un objectif. Et le projet est défini comme un ensemble hiérarchisé d'objectifs conscients ou non. Bien entendu, agir de manière appropriée requiert de tenir compte des contraintes auxquelles tout un chacun est soumis.

L'ensemble de ces contraintes définit la situation de l'acteur. L'action vise à adapter la situation aux objectifs. Mais l'adaptation est à double sens : l'acteur doit aussi choisir ses objectifs, et donc les adapter en fonction de sa situation, sous peine autrement de choisir des objectifs utopiques. Enfin, ce qui compte en dernière instance c'est la perception qu'a l'acteur de ses objectifs et de sa situation. Les premiers sont parfois vagues et implicites; ils sont réexaminés à la lumière des difficultés rencontrées par les acteurs dans la mise en œuvre de leurs décisions. Quant à la situation, elle n'est jamais parfaitement connue dans tous les détails. C'est en agissant que l'acteur prend mieux conscience des contraintes de sa situation. Il modifie alors sa perception de sa propre situation.

Ainsi réduit à ses composantes essentielles, le modèle peut paraitre très/trop abstrait. Mais comme tout modèle théorique, son intérêt réside dans sa capacité à permettre "l'analyse concrète des situations concrètes ». Autrement dit, tout dépend de sa validation empirique. La publication de synthèse citée ci-dessus donne de nombreuses références d'analyses empiriques conduites dans les années 1960 et 1970 dans diverses régions françaises et à l'étranger, présentées comme des validations empiriques de la théorie du comportement adaptatif.

Deux caractéristiques distinguant la TCA de la théorie néoclassique de la production méritent d'être soulignées ici : d'une part, le caractère exogène des fonctions de production et d'utilité y est remis en cause, ou plutôt dépassé et, d'autre part, elle a une dimension dynamique dépassant le caractère statique de la théorie néoclassique. Concernant la fonction de production, la TCA ne prétend pas expliquer les lois techniques de la production mais, en mettant l'accent sur la hiérarchie des actions et des sous-actions, elle conduit à souligner l'importance des pratiques productives ; celles-ci doivent être distinguées des techniques ${ }^{9}$. Leur choix fait l'objet de décisions qui peuvent être interprétées dans le même cadre théorique en termes de projet et de situation. Quant à la fonction d'utilité, elle est remplacée par la structure hiérarchisée des objectifs ou des finalités, dont on a vu qu'ils étaient euxmêmes interprétés comme résultant de processus permanents d'adaptation (Fig. 1). Enfin, la nature dynamique de la théorie du comportement adaptatif est essentielle : l'acteur/décideur s'adapte en permanence, ce qui permet des interprétations fines du comportement des agriculteurs, aussi bien dans leurs décisions quotidiennes que dans leurs choix stratégiques à plus long terme, comme ceux d'adoption des innovations techniques. La TCA a été une sorte d'objet intermédiaire dans le travail interdisciplinaire d'analyse du fonctionnement des exploitations agricoles

9. J.-H. Teissier (1979) a le premier souligné l'importance de cette distinction et suggéré une explication des relations entre techniques et pratiques. 
(Vinck, 2009). En effet, elle a permis de définir un objet commun qui était les "pratiques » des agriculteurs.

Soulignons enfin que cette théorie doit beaucoup à diverses disciplines de sciences sociales telles que la sociologie, l'épistémologie, la psychologie, etc. comme l'illustre la diversité des auteurs cités dans les articles référencés ci-dessus comme par exemple Rogers, Simon, Bachelard, ou Piaget. C'est avec les sciences de gestion que les relations ont été les plus étroites. Rappelons d'ailleurs que l'économie rurale américaine, au sein de laquelle a été formé l'un d'entre nous, est née au début du XXe siècle de la collaboration des économistes avec les spécialistes de la gestion des exploitations agricoles (farm management). Plus tard, les collaborations avec les " gestionnaires " des centres de recherche en gestion de l'École Polytechnique (CRG), de l'École des Mines (CGS), de l'Institut d'administration des entreprises (IAE) ont été nombreuses. Par ailleurs, signalons la proximité de la notion de perception, centrale dans la théorie du comportement adaptatif, avec le concept de représentation élaboré par les psychologues, Piaget en particulier, et vulgarisé par les psycho-sociologues d'entreprise. Enfin, l'accent mis sur les pratiques a invité aux rapprochements avec l'anthropologie et a facilité les collaborations étroites avec les sciences techniques (agronomie et zootechnie, en particulier...).

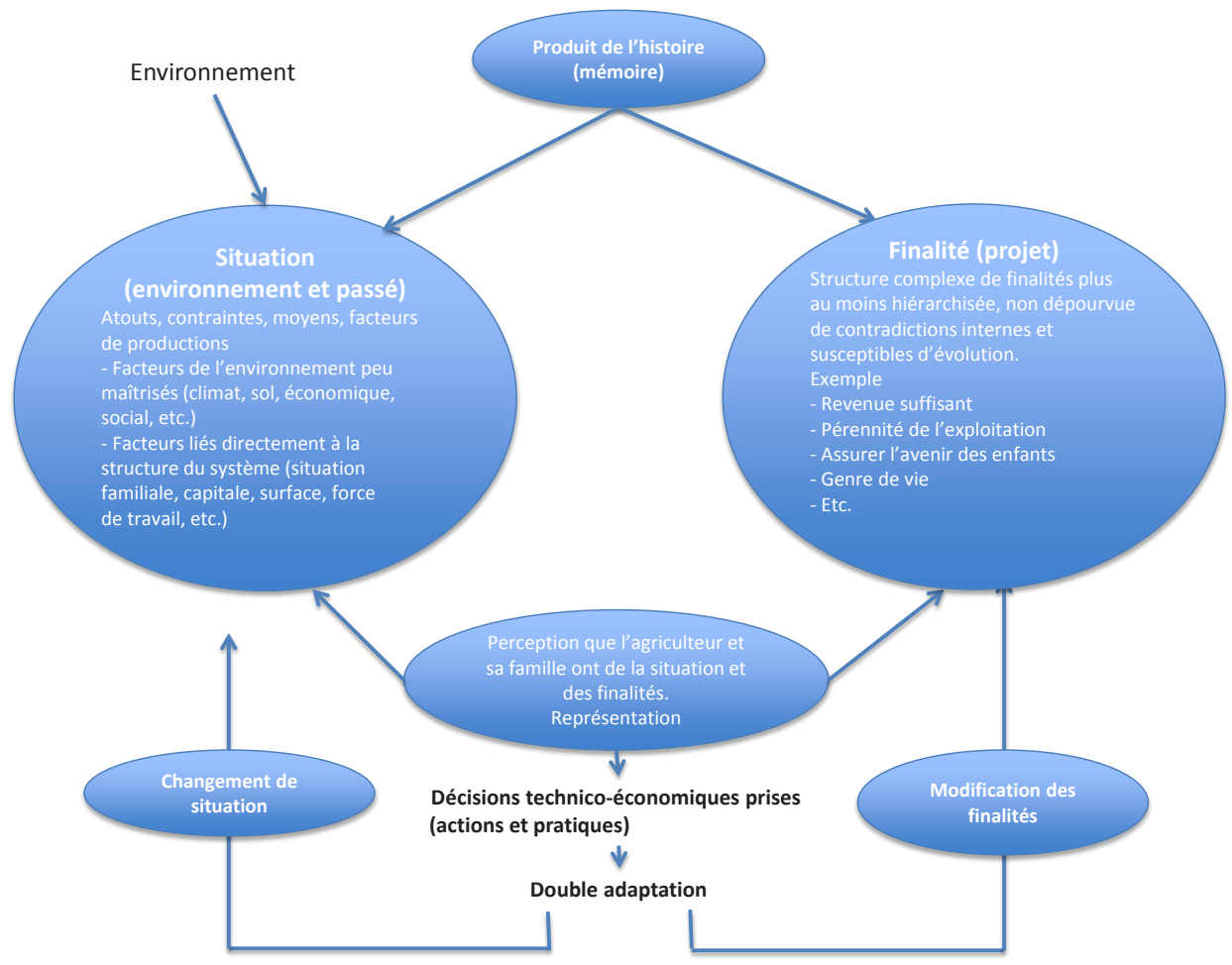

Fig. 1. Modèle du comportement adaptatif du système famille exploitation (source : Brossier et al., 1997). 


\section{La TCA comme référence théorique utile}

Rétrospectivement, on voit bien que la TCA est apparue à un moment précis dans l'histoire de l'agriculture française, moment qui correspondait à un besoin de renouvellement de la théorie pour comprendre le comportement des agriculteurs, en particulier leur plus ou moins grande propension à adopter le progrès technique (Petit, 1975). Depuis la fin de la Seconde Guerre mondiale, il était généralement admis que les agriculteurs devaient naturellement adopter le progrès technique sans état d'âme, tellement ce progrès était considéré comme efficace et supérieur à la situation antérieure. Il devait permettre aux agriculteurs de " maximiser le profit "! À l'époque, tout un courant de pensée fleurissait, qualifiant les agriculteurs de retardataires, passéistes, voire archaïques pour " expliquer » leur refus du progrès technique. L'objectif avoué des politiques publiques et des actions collectives professionnelles était de transformer l'agriculture en une activité vraiment industrielle, pour la sortir du passé et la mettre à l'heure moderne : il fallait donc transférer vers le monde agricole les outils industriels (en particulier la comptabilité), faire en sorte que les agriculteurs changent de rationalité pour qu'ils adoptent celle du monde industriel et qu'ils deviennent ainsi des entrepreneurs! La nécessité de l'industrialisation de l'agriculture est d'ailleurs explicitement affirmée par J. Chombart de Lauwe dans son manuel cité précédemment, publié seulement quelques années avant nos premiers travaux.

Nous illustrons, ici, la pertinence de la théorie du comportement adaptif à cette époque sur trois points : le renouvellement du regard sur les agriculteurs familiaux impliqués dans la modernisation de l'agriculture en France dans les années 1970, sa contribution à la rénovation pédagogique de l'enseignement technique agricole en France également et le passage d'une conception de la gestion centrée sur des outils normatifs à une conception centrée sur l'acteur et son projet, valable aussi au-delà de la France. Enfin, pour illustrer la pertinence de cette théorie pour l'éclairage des politiques publiques, nous décrirons brièvement sa contribution à la définition des mesures d'aide aux " agriculteurs en difficulté " dans les années 1980. Soulignons que cette pertinence repose sur la capacité de la TCA de permettre des analyses à la fois fines et complètes du fonctionnement technico-économique des exploitations agricoles.

\section{Renouvellement du regard sur les agriculteurs impliqués dans la modernisation de l'agriculture en France dans les années 1970}

Quelques chercheurs, de l'Inra et d'autres organismes, et des techniciens, proches du monde agricole, s'appuyant sur leurs pratiques et une observation fine des situations concrètes, ont peu à peu remis en cause le postulat de non-rationalité économique des paysans et contesté ce modèle trop simpliste. Ils ont finalement suggéré que 


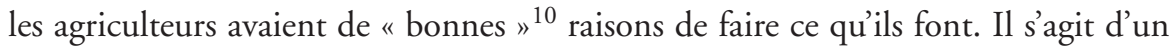
postulat et non d'une hypothèse, car on ne peut pas vraiment le remettre en cause : l'analyste doit postuler qu'il y a toujours une explication aux comportements qu'il observe. Très rapidement, il est apparu qu'une des explications majeures de cet écart entre les décisions des agriculteurs et la rationalité économique dominante était liée au caractère encore essentiellement familial de la plupart des exploitations agricoles après la deuxième guerre mondiale. La prise en compte des rapports étroits existant entre la famille et l'exploitation agricole (en considérant qu'ils faisaient ensemble un système) expliquait des comportements qui apparaissaient ainsi tout à fait cohérents. Et il est vrai que pendant les premières décennies après la guerre, le caractère familial avait plutôt tendance à se renforcer avec la forte diminution du salariat en agriculture et donc l'augmentation de la part familiale dans le travail ${ }^{11}$.

Il faut enfin souligner que l'élaboration de cette théorie s'est faite alors que les auteurs, essentiellement des économistes, étaient étroitement impliqués dans des équipes pluridisciplinaires d'agronomes, incluant des agronomes stricto sensu et des zootechniciens ${ }^{12}$. Ces chercheurs faisaient notamment partie de l'équipe VersaillesDijon, devenue plus tard l'équipe Versailles-Mirecourt-Dijon, l'une des structures de l'Inra à partir de laquelle a été fondé le département systèmes agraires et développement ${ }^{13}$ en 1979 (voir Brossier et al 1994 pour une présentation en anglais des travaux du Sad). Ces chercheurs ont élaboré et défendu quelques idées-forces :

- l'exploitation agricole doit être vue comme un système finalisé, comme l'a souligné Pierre-Louis Osty dans son article séminal (Osty, 1978), c'est-à-dire " qu'il faut considérer d'abord l'ensemble avant d'étudier à fond les parties que l'on sait aborder ", car l'exploitation est un tout organisé. Ils retrouvent ainsi les intuitions de Pascal : « Je tiens pour impossible de connaitre le tout sans connaitre les parties et de connaitre les parties sans connaître le tout ";

- l'exploitation est donc en fait un système appelé système exploitation famille (SEF). Elle doit être vue comme un centre de décision en postulant que « les agriculteurs ont des raisons de faire ce qu'ils font ", postulat assez novateur et contesté à l'époque, qui s'est avéré féconde ;

10. Nous avons eu un débat intense et important pour savoir s'il s'agissait de «bonnes » raisons, terme que nous employons dans nos premières communications. Nous avons peu à peu supprimé le terme " bonnes " qui pouvait être mal interprété. En fait par "bonnes " nous voulions souligner que les agriculteurs prenaient des décisions qui leur semblaient bonnes pour eux, c'est-à-dire cohérentes compte tenu de l'état de leurs connaissances et des informations qu'ils possédaient. C'est sur ces informations, plus ou moins complètes ou adéquates, qu'il pouvait y avoir débat et possibilité d'agir, notamment par la formation.

11. Aujourd'hui, ce caractère familial diminue à nouveau, non pas que les exploitations ne soient pas gérées par des familles, mais du fait qu'il y a souvent deux activités différentes au sein du couple d'agriculteurs, où s'exprime de plus en plus le besoin de bien séparer les deux activités.

12. Citons nos collègues J.-P. Deffontaines, P.-L. Osty, Y. Houdard, J.-H. Teissier, J. Bonnemaire et $\mathrm{M}$. Roux à Versailles et à Dijon, et aussi C. Béranger et G. Liénard à Clermont-Ferrand.

13. Aujourd'hui Sad signifie Sciences pour l'action et le développement. 
- l'exploitation agricole est une exploitation familiale ;

- le comportement des agriculteurs est adaptatif (TCA);

- les pratiques sont devenues un objet d'étude qui permet non seulement de comprendre la rationalité des agriculteurs mais aussi d'analyser le fonctionnement des exploitations.

Ces résultats ont été traduits et transformés en connaissances enseignables, dans les programmes de l'enseignement agricole tant au niveau des lycées et des centres de formation technique, que des écoles d'ingénieurs. Cette activité ${ }^{14}$ était constitutive de nos recherches ainsi que de notre posture d'investigation. Dans le paragraphe suivant, nous examinons l'apport de la TCA à l'enseignement et réciproquement car l'enseignement nous a aussi beaucoup apporté en particulier dans la formalisation de nos résultats pour notamment les rendre enseignables.

\section{L'influence de la TCA dans la rénovation pédagogique de l'enseignement technique agricole}

Ces résultats ont suscité un écho fort auprès de ceux qui avaient à animer la rénovation pédagogique de l'enseignement agricole ${ }^{15}$. Pour la formation des agriculteurs, la principale question pédagogique qui s'imposait était alors : comment passer des connaissances, essentiellement de nature disciplinaire, aux compétences?

Plus précisément :

- comment dépasser les approches sectorielles de l'exploitation c'est-à-dire l'énoncé par chaque discipline de codes de bonne conduite qui se préoccupent peu de leur applicabilité en univers contraint ?

- comment passer d'une pédagogie centrée sur les contenus à une pédagogie qui se donne pour objectif une préparation à l'action de l'agriculteur ?

Une dynamique de collaboration scientifique s'engage alors entre la recherche pédagogique et la recherche agronomique par l'intermédiaire des ingénieurs d'agronomie de l'Inrap. Ces ingénieurs ont alors l'intuition que le renouvellement de la formation des agriculteurs passe en particulier par la mise au point d'une méthode pédagogique rigoureuse fondée sur une approche globale de l'exploitation agricole, que nous préconisions. Cela se traduira par la publication d'ouvrages pédagogiques sur l'approche globale du fonctionnement de l'exploitation agricole (Bonneviale et al., 1989 et Marshall et al.,1994) qui, tout comme l'ouvrage de synthèse de Brossier

14. Rappelons que plusieurs membres de l'équipe de recherche de Dijon étaient des enseignants. 15. La principale institution impliquée était l'Institut national de recherche et d'applications pédagogiques (Inrap) installée également à Dijon, où trois ingénieurs d'agronomie (R. Bonneviale, R. Jussiau et E. Marshall), chargés respectivement des disciplines agronomie, zootechnie et économie rurale, sont devenus nos interlocuteurs privilégiés. La relation avec Éric Marshall, membre de notre équipe de recherche, a été très étroite pendant de longues années. 
et al. (1997) déjà cité, servent encore de «text book» pour la formation dans les lycées agricoles $^{16}$.

\section{D'une conception de la gestion centrée sur des outils normatifs à une conception centrée sur l'acteur et son projet}

Suite à nos travaux sur les pratiques des agriculteurs, nous avons proposé une méthode de diagnostic (Benoit et al., 1988), fondée sur une investigation technicoéconomique fine des différentes fonctions via les pratiques. Ce diagnostic montre d'abord à l'agriculteur qu'il est possible d'analyser et de porter un jugement sur son exploitation en tenant compte de sa propre perception. Il est donc possible pour l'agriculteur de s'approprier lui-même les outils de gestion, c'est à dire d'être en mesure de mobiliser son savoir spontané en y associant le savoir externe. Plusieurs outils ont été élaborés autour de l'approche globale évoquée ci-dessus, enrichie des relations scientifiques que nous avons maintenues de longue date avec les socioéconomistes d'entreprises du CRG et du CGS ${ }^{17}$, et avec J.-L. Le Moigne, théoricien fécond de la modélisation systémique (Le Moigne, 1977,1990 ; Brossier, Vissac et Le Moigne, 1990).

Notre démarche nous a rapprochés des courants de la recherche-action (Liu, 1992), de la recherche intervention (Girin, 1986) ou de la recherche clinique (Chia, 1992) qui se développaient dans plusieurs champs scientifiques à cette époque. Ces nouvelles approches nous ont aidés à développer notre conception de la gestion. Nous avons été souvent amenés - en relation avec la demande de groupes d'agriculteurs qui s'interrogeaient sur leurs choix techniques, sur l'évolution de leurs systèmes de production et sur leurs grandes orientations stratégiques - à mettre au point une méthode expérimentale s'appuyant sur les relations entre recherche, formation et action pour l'analyse des décisions. Observer les pratiques des agriculteurs dans une perspective de gestion a supposé cette méthodologie particulière, proche de la démarche clinique pour favoriser cette maïeutique ou «l'art d'accoucher les esprits ». Ce fut l'occasion de l'orientation décisive de notre équipe vers la gestion.

Des groupes d'agriculteurs avec qui nous avons travaillé dans les années 1970 (en Haute-Saône, dans le Choletais, dans le Chatillonnais, ou encore à Beaune, à Neufchâteau) ont été de véritables laboratoires socio-économiques, lieux privilégiés d'étude de l'action et du comportement économique, des agriculteurs. Ces agriculteurs-participants étaient engagés dans un processus de changement dans leurs exploitations. Ces différents cas d'étude nous ont permis d'étudier la relation

16. Il faut aussi citer l'ouvrage pédagogique coédité par Nathan et Educagri, sous la direction d'Éric Marshall (Marshall et al., 1998) qui présente l'exploitation agricole comme un système complexe, la TCA y étant présentée comme un outil théorique d'aide à la décision.

17. Ce lien avec ces économistes (Berry et al., 1979, Riveline, 1988) nous a été très utile pour asseoir notre position qui pouvait apparaître comme trop agriculturo-centrée. 
entre réflexion et action, en nous basant principalement sur la simulation des changements, grâce notamment à l'outil de programmation linéaire, fournissant une base de discussion pour supputer et discuter les conséquences tant économiques qu'organisationnelles des changements envisagés. En effet, ces recherches-actions, sur les décisions des agriculteurs, visaient à la fois la formation à la gestion des agriculteurs du groupe et le changement dans leurs exploitations.

\section{Quelles mesures pour aider les " agriculteurs en difficulté " dans les années 1980 ?}

L'attention politique portée aux " agriculteurs en difficulté » est apparue avec force à la fin des années 1980. Le défi pour la recherche était non seulement d’identifier, de caractériser et de quantifier le phénomène mais aussi, comme le demandait le ministère de l'Agriculture, de fournir des critères permettant la " détection précoce ", le suivi et l'accompagnement de ces agriculteurs dans le processus de redressement. Nos travaux sur les pratiques de trésorerie des agriculteurs avec la thèse de l'un d'entre nous (Chia, 1987) qui mettaient l'accent sur l'importance de bien connaître ces pratiques pour comprendre le fonctionnement des exploitations ont permis d'une part, de valider le principe d'adaptation (de la situation au projet et du projet à la situation) de la théorie du comportement adaptatif, et d'autre part de proposer des critères à la fois simples, quantitatifs et compris de tous (langage commun), comme le "taux d'endettement immédiat " (total de remboursement d'emprunt/ total de ventes) ou " la pression familiale » (total des dépenses familiales/total de ventes) pour détecter précocement les situations de fragilité financière (Colson et al., 1993). Ces travaux ont permis aussi d'identifier les mécanismes d'adaptation et de décisions des agriculteurs en cas de difficultés financières. Un des premiers postes de dépenses sur lequel les agriculteurs agissaient était les prélèvements privés. Venaient ensuite les frais de cotisation à la MSA (mutualité sociale agricole), pour les assurances grêles, incendies... puis pour les éleveurs, la réduction des dépenses d'alimentation pour le bétail et pour les agriculteurs, les dépenses d'engrais. Nous avons constaté que dans certains cas, si l'on avait appliqué les critères issus du modèle comptable, ces exploitations auraient dû disparaître depuis très longtemps. Or, elles avaient trouvé des moyens de s'adapter à leur situation très difficile leur permettant ainsi de survivre.

\section{Prolongements et ouvertures}

La théorie du comportement adaptatif nous semble encore pertinente aujourd'hui. Mais comment étayer cette conviction ? Et comment préciser sur quoi cette pertinence repose ? Nous répondons à la première question dans cette partie et discutons de la seconde dans la conclusion. 
Deux prolongements méritent ainsi d'être soulignés ici : l'un est d'ordre géographique, l'autre thématique. D'une part, la TCA a eu une résonance internationale et s'est révélée pertinente dans des contextes très différents de l'agriculture familiale française des années 1970, période durant laquelle elle a été développée. D’autre part, elle a aussi été utile pour guider les premiers travaux sur la flexibilité des exploitations en France il y a une dizaine d'années.

\section{Prolongement international et pertinence actuelle de la TCA dans les pays du Sud : particulièrement en Afrique et Amérique Latine}

Au plan international, une des dernières publications s'inspirant directement des travaux sur la TCA est l'ouvrage codirigé par J. Brossier et B. Dent, Gestion des exploitations et des ressources rurales, entreprendre, négocier, évaluer (1998) qui rassemble des contributions internationales. Il faut aussi citer l'activité de la branche européenne de l'IFSA (International Farming Systems Association) qui, depuis près de 20 ans, a continué à organiser des colloques biennaux (le XXI e aura lieu en 2015) où les travaux, en particulier ceux du Sad, sur la petite exploitation familiale ont continué à recevoir une bonne audience ${ }^{18}$.

En Afrique et en Amérique latine, comme dans beaucoup d'endroits dans le monde, la majorité des exploitations sont familiales et ce caractère familial n'est pas prêt de disparaître. Ce fait explique probablement que l'approche basée sur la TCA ait pu recevoir une reconnaissance notable, et qu'elle ait servi de référence pour aider à comprendre le fonctionnement des exploitations dans un contexte pourtant très différent de celui des exploitations agricoles familiales françaises des années 1970. Les analyses qui s'en sont inspirées ont aussi permis de proposer des actions de développement plus adaptées au contexte local que celles s'inspirant d'approches plus classiques en économie. La TCA a donc eu un certain retentissement et une bonne diffusion en Afrique, en particulier francophone, et en Amérique latine grâce notamment aux étudiants africains et latino-américains formés à Dijon. Elle a également été utilisée par les collègues du Cirad pour comprendre et proposer des actions de développement (Gafsi et al., 2007). De plus, le développement en Afrique de la démarche "conseil de gestion ", promue par la FAO avec le soutien de la Banque mondiale et auquel le Cirad a été étroitement associé, a beaucoup contribué à la diffusion des concepts et outils associés à la TCA. Ce rayonnement est illustré par quelques publications dont deux ouvrages de synthèse ${ }^{19}$.

18. Voir en particulier l'ouvrage synthétique que le département Sad a produit pour présenter ses travaux lors du symposium international de l'IFSA en 1994 (Brossier et al., 1994).

19. En 2006, le numéro spécial des Cahiers d'Agriculture (Chia, Dugué, 2006) intitulé « L'exploitation agricole familiale : mythe ou réalité ? » qui s'adresse aux agricultures du monde est une illustration du maintien de la pertinence de ces travaux.

Fin 2007, un ouvrage de synthèse largement diffusé en Afrique via l'ACTA (co-financeur) a pour titre : Exploitations agricoles familiales en Afrique de l'ouest et du centre (Gafsi et al. 2007). 


\section{Flexibilité des exploitations agricoles familiales}

Force est de constater que les recherches en gestion ont peu abordé la flexibilité des entreprises agricoles. La TCA a inspiré nos travaux sur les pratiques de trésorerie des agriculteurs (Chia, 1987, 1992), où nous avions développé un concept voisin : la " capacité de négociation ", définie comme la capacité des agriculteurs à faire face aux changements internes et externes. Elle est fondée sur trois types de capitaux : culturel, social et économique.

A la fin des années 1990, les agriculteurs avaient traversé plusieurs situations difficiles (baisse de prix de produits, mauvaises années...) et la crise de la "vache folle " avait non seulement eu comme conséquence une baisse du prix de la viande bovine, suite à une diminution de la demande, mais avait aussi touché la confiance que les consommateurs portent aux agriculteurs (remise en cause du métier). Lors de nos travaux sur les impacts de la crise de la vache folle, nous avons constaté qu'un certain nombre d'éleveurs s'en sortaient mieux que d'autres. Certains vendaient leurs animaux en broutards pour le marché italien, d'autres, au contraire, gardaient leur troupeau au complet, d'autres cherchaient à diminuer le nombre d'intermédiaires dans leurs circuits de commercialisation. On avait affaire à une diversité de pratiques, pratiques qui correspondaient à des situations différentes et à des projets différents. Ces pratiques traduisaient le mécanisme d'adaptation entre situation et projet. Nous avons émis l'hypothèse qu'en étudiant la flexibilité des exploitations agricoles, on pouvait accéder à la gamme très variée des mécanismes d'adaptation.

Nous avions constaté également l'importance de l'action collective dans les stratégies à l'œuvre. Les enjeux se situent alors à la fois au niveau des unités de production et au niveau de l'organisation collective : il s'agissait donc pour nous de caractériser et de comprendre les nouveaux modes de gestion des exploitations d'élevage dans leurs différentes dimensions (techniques, économiques, organisationnelles et sociales).

Aujourd'hui, compte tenu de l'incertitude à laquelle les exploitations doivent faire face, l'action individuelle n'est plus suffisante et l'action collective est devenue le principal levier d'action. Ceci constitue la principale caractéristique de la flexibilité. Il s'agit essentiellement d'une "flexibilité relationnelle" (Dedieu et al., 2008). Elle s'exprime surtout à travers la participation des producteurs à des réseaux, des groupements, des coopératives et des associations. Dans le cas des éleveurs, par exemple, en se regroupant, pour la commercialisation de leurs animaux, ils améliorent leur capacité de négociation. En Argentine, la pratique de "capitalización " permet de rester producteur en élevant et en engraissant sur ses propres terres des animaux appartenant à d'autres. Mais cette pratique n'est possible que parce que les éleveurs participent à des réseaux. Les travaux sur la flexibilité ont montré l'importance des mécanismes d'adaptation dans le maintien et la pérennisation des exploitations. Ce faisant, ils ont démontré une nouvelle fois la pertinence de la TCA, en tant qu'outil

La théorie du comportement adaptatif y est présentée largement (deux chapitres portent sur la présentation de la théorie) et est illustrée par quelques articles. 
d'analyse et de modèle d'action : le projet s'adapte à la situation par le biais des perceptions, qui elles-mêmes changent en s'adaptant.

\section{Conclusion : perspectives d'avenir}

Les propos qui précèdent, notamment ceux sur la flexibilité des exploitations, illustrent la pertinence actuelle de la théorie du comportement adaptatif. Ce constat peut paraître paradoxal alors que, on l'a vu, l'élaboration de la TCA correspond à un moment bien précis de l'évolution de l'agriculture française, moment où l'exploitation agricole familiale, d'un type bien spécifique, joue un rôle crucial à la fois dans les faits, dans les discours et dans la conception des politiques publiques, comme l'illustre la place centrale donnée à l'exploitation à deux UTH (unités de travail humain) dans les lois d'orientation des années 1960. Selon nous, ce paradoxe s'explique par le fait que la TCA est fondamentalement une théorie microéconomique très générale.

Les deux caractéristiques, théorique et microéconomique, méritent attention. Comme toute théorie, il s'agit bien sûr d'une représentation simplifiée de la réalité. Par exemple, les comportements humains n'apparaissent pas toujours rationnels mais l'analyste est bien obligé, pour expliquer les comportements, de postuler que les hommes et les femmes ont des raisons de faire ce qu'ils font ; et un objectif de la recherche est précisément de trouver quelles sont ces raisons. Ceci permet de dépasser l'hypothèse contraignante de la recherche du profit maximum et d'incorporer, par exemple, des objectifs relatifs à la gestion du patrimoine. En fin de compte, l'observation montre, croyons-nous, qu'en matière de gestion des unités de production agricoles, il est possible de rendre compte des décisions du producteur en analysant la dialectique projet/situation.

Les implications du caractère micro-économique de cette théorie sont peut-être plus subtiles et moins généralement perçues. En effet, privilégier le niveau micro, même si ce n'est que pour une étape analytique dans une construction intellectuelle plus ambitieuse, est peut-être une démarche interdite lorsqu'on travaille sur l'action collective où l'individu est toujours appréhendé comme être social, que l'on ne peut pas isoler des relations sociales dans lesquelles il est inséré. Or, comme indiqué dans la première partie, la TCA est issue directement de la théorie économique de la production, où le niveau microéconomique joue un rôle crucial. Et c'est précisément ce niveau microéconomique qui donne à la TCA son caractère général, susceptible d'épouser les différentes formes d'agriculture familiale. En effet, la TCA invite à explorer le choix des objectifs d'action en fonction de l'analyse fine de la situation. Et justement, les situations varient selon les différentes formes d'agriculture familiale. Pour prendre un exemple français, le fait que la place des femmes ait radicalement changé dans la société et, par suite, dans l'agriculture a bouleversé la dialectique projet/situation dans la plupart des exploitations. Ceci est tout à fait compatible avec la TCA et celle-ci peut aider à analyser de tels bouleversements. 
Mais il serait naïf d'ignorer un danger de ce caractère de théorie microéconomique générale. Reposant sur le principe de l'individualisme méthodologique, ignorant ainsi le contexte social, privilégiant de fait les relations marchandes de l'unité de production avec son environnement économique, la TCA a pu être utilisée de façon apologétique pour défendre un certain modèle d'exploitation agricole familiale, jouant un rôle idéologique important dans les débats sociaux et politiques des années 1970. Or ce point de vue ignorait totalement des aspects très négatifs de ce modèle, tels que ceux portant sur la place mineure donnée aux femmes, comme l'ont montré les travaux de Alice Barthez, sociologue, durant toute sa carrière (Barthez, 2005). On retrouve bien là, la principale limite de la TCA soulignée ci-dessus : parce qu’il s'agit d'une théorie microéconomique, elle ne peut pas seule rendre compte des changements de structure économique et sociale.

En revanche, le niveau microéconomique a le grand avantage de permettre des analyses fines de situations individuelles, susceptibles de renouveler et d'approfondir des analyses plus générales. Ceci sera illustré ici sur la nature plus ou moins familiale des grandes exploitations agricoles du bassin parisien. À bien des égards celles-ci peuvent être vues comme des exploitations capitalistes. Tel a été le point de vue exprimé par de nombreux auteurs depuis longtemps. Pourtant, nous avions conclu notre recherche sur les " régions de grande culture " il y a quarante ans en soulignant la nécessité de prendre en compte « le caractère familial de l'appropriation des moyens de production, de la constitution et de la gestion du patrimoine pour apprécier l'évolution de ce secteur " (Brossier et al., 1974). Et nous pensons que ce résultat reste encore largement valable aujourd'hui ! Cette différence d'appréciation n'est pas citée ici pour dénoncer l'erreur supposée de telle ou telle classification. Notre propos est de souligner l'apport possible d'une analyse fine au niveau microéconomique qui permet de nuancer un point de vue basé sur une perspective plus globale ou, plus précisément de porter un jugement critique sur un tel point de vue. C'est bien le modèle théorique microéconomique qui nous a permis de remettre en cause l'idée assez généralement admise que l'agriculture du bassin parisien était un exemple d'agriculture capitaliste. Nous croyons avoir ainsi enrichi la gamme reconnue des formes très diverses d'agriculture familiale. Dans cette perspective, on peut d'ailleurs considérer que l'agriculture du Middle West américain est largement restée de type familial, par certains aspects au moins, alors que, par exemple, les très grandes exploitations d'Ukraine, de Russie ou du Kazakhstan, héritières des anciens sovkhozes soviétiques, sont de nature très différente.

Au total, la TCA se présente aujourd'hui encore comme un outil théorique utile. Située délibérément et exclusivement au niveau microéconomique, focalisée sur les décisions des agriculteurs, elle est précieuse comme cadre de recherches pluridisciplinaire impliquant les agriculteurs comme participants à part entière. C'est donc un instrument utile pour organiser la combinaison des savoirs scientifiques et des savoirs pratiques, formels et informels des acteurs. On sait combien de telles combinaisons sont à la fois nécessaires aujourd'hui et difficiles à réaliser. 


\section{Bibliographie}

Barthez, A., 2005. Devenir agricultrice : À la frontière de la vie domestique et de la profession, Économie Rurale, 289-290, 35-45.

Becker, G., 1965. A Theory of the Allocation of Time, Economic Journal 75, 299, 493-517.

Benoît, M., Brossier, J., Chia, E., Marshall, E., Roux, M., Morlon, P., Teilhard de Chardin, B., 1988. Diagnostic global d'exploitation agricole : une proposition méthodologique, Études et recherches sur les systèmes agraires et le développement, 12 .

Berry, M., Moisdon, J.-C., Riveline, C., 1979. Qu'est-ce-que la recherche en gestion? Informatique et gestion, 108, 109.

Bonneviale, J.-R., Marshall, E., Jussiau, R., 1989. Approche globale de l'exploitation agricole. Comprendre le fonctionnement de l'exploitation agricole : une méthode pour la formation et le développement, Inrap Foucher.

Bradford, L.A., Johnson, G.L., 1953. Farm Management Analysis, New York, London, John Wiley and Sons.

Brossier, J., de Bonneval, L., Landais, E., (Ed) 1994. Systems Studies in Agriculture and Rural Development, INRA Science Up to date, 300 p.

Brossier, J., Brun A., Deffontaines, J.-P., Fiorelli, J.-L., Osty, P.-L., Petit, M., Roux, M., 2008. Quels paysages avec quels paysans? Les Vosges du Sud à trente ans d'intervalle, Versailles, éditions Quae.

Brossier, J., Dent, B. (Ed). 1998. Gestion des exploitations et des ressources rurales : entreprendre, négocier, évaluer, Farm and Rural Resources management : New Context, New Constraints, New Opportunities. Coordination scientifique et introduction du numéro, Études et recherches sur les systèmes agraires et le développement, 31.

Brossier, J., Marshall, E., Chia, E., Petit, M., 1997. Gestion de l'exploitation agricole familiale. Eléments théoriques et méthodologiques, Educagri éditions/ENESAD/ CNERTA (Nouvelle édition 2002).

Brossier, J., de la Vaissiere, P., Lienard, G., Petit M., 1974. Systèmes de production en région de grandes cultures, Série Économie et Sociologie Rurales, Inra Versailles.

Brossier, J., Vissac, B., Le Moigne, J.-L., (Eds), 1990. Modélisation systémique et système agraire. Décision et organisation, Actes du Séminaire du Département SAD, Saint-Maximin (2-3 Mars 1989), Versailles, INRA-SAD.

Chia, E., 1987. Les pratiques de trésorerie des agriculteurs. La gestion en quête d'une théorie, Thèse de doctorat, Faculté de Sciences économiques et de gestion de Dijon.

Chia, E., 1992. Une "recherche clinique ": proposition méthodologique pour l'analyse des pratiques de trésorerie des agriculteurs. Étude de cas en Lorraine, Études et recherches sur les systèmes agraires et le développement, 26.

Chia, E., Dugué, P., (Coord.), 2006. L'exploitation agricole familiale : mythe ou réalité ? Cahiers d'Agriculture, Numéro spécial, 15, 6. 
Chombart de Lauwe, J., Poitevin, J., Tirel, J.-C., 1963. Nouvelle gestion des exploitations agricoles, Paris, Dunod.

Colson, F., Blogowsky A., Dechambre B., Chia E., Desarmenien D., Dorin B., 1993. Prévenir les défaillances financières en agriculture - Application de la méthode des scores INRA, Cahiers d'Économie et Sociologie Rurales, 29, 21-44.

Dedieu, B., Chia, E., Leclerc, B., Moulin, C.H., Tichit, M., 2008. L'élevage en mouvement - Flexibilité et adaptation des exploitations d'herbivores, éditions Quae.

Dillon, J., 1971. An Expository Review of Bernoullian Decision Theory in Agriculture : Is Utility Futility ? Review of Marketing and Agricultural Economics, 39, 1.

Gafsi, M., Dugué, P., Jamin, J.-Y., Brossier, J., (Eds) 2007. Exploitations agricoles familiales en Afrique de l'Ouest et du Centre : enjeux, caractéristiques et éléments de gestion, Versailles, éditions Quae.

Girin, J., 1986. L'objectivation des données subjectives. Éléments pour une théorie du dispositif dans la recherche interactive, in Actes du colloque FNEGE-ISEOR: Qualité des informations scientifiques en gestion. Méthodologies fondamentales en gestion, 18-19 novembre 1986, 170-186.

Guillou, M. 2013. Le projet agroécologique : vers des agricultures doublement performantes pour concilier compétitivité et respect de l'environnement, Rapport présenté le 11 juin 2013 à Stéphane Le Foll, ministre de l'Agriculture, de l'Agroalimentaire et de la Forêt.

Hervieu, B., Purseigle, F., 2013. Sociologie des mondes agricoles, Paris, Armand Colin. Johnson, G.L., Halter, A.N., Jensen, H.R., Thomas, D.W., 1961. A Study of the Managerial Processes of Midwestern Farmers, Ames, Iowa State University Press.

Le Moigne, J.-L., 1977. La théorie du système général, théorie de la modélisation, paris, PUF (nouvelle édition, 1990).

Liu, M. 1992. La Recherche-Action, Revue Internationale de Systémique, 6, 4 Numéro spécial.

Marshall, E., Bonneviale, J.-R., Francfort, I., 1994. Fonctionnement et diagnostic global de l'exploitation agricole; une méthode interdisciplinaire pour la formation et le développement, Educagri (nouvelle édition 2013).

Marshall, E., Bonneviale, J.-R., Brossier, J., Ferrié, H., Fremont, H., Schost, C., Vincq, J.-L., 1998. L'exploitation agricole (histoire, organisations sociales, production, diversité, gestion), Dijon, Coédition Nathan/Educagri, collection "Repères Pratiques ".

Nerlove, M., 1974. Household and Economy : Toward a New Theory of Population and Economic Growth, in Theodore, W., Schultz (Ed) Marriage, Family, Human Capital, and Fertility, Chicago, University of Chicago Press, 200-221.

Osty, P.-L., 1978. L'exploitation vue comme un système, Bulletin Technique d'Information 326, 43-49.

Petit, M., 1975. Farmers' adoption of technical innovations, European Review of Agricultural Economics, 3 (2/3).

Riveline, C., 1988. Lévaluation des coûts, Annales des Mines, Première édition Juin 1973. 
Schultz, T.W., 1972. The Increasing Economic Value of Human Time, American Journal of Agricultural Economics, 54, 5, 843-850.

Sotomayor, O., Rodriguez A., Rodrigues M, 2011. Competitividad, sostenibilidad e inclusión social en la agricultura : una mirada hacia América Latina y el Caribe 2014. Nuevas direcciones en el diseño de políticas en América Latina y el Caribe, [en ligne] http://www.eclac.org/publicaciones/xml/8/45598/2011593-LBC-113_WEB.pdf

Teissier, J.-H., 1979. Relations entre techniques et pratiques, Bull. Inrap, Dijon, 38.

Vinck, D., 2009. De l'objet intermédiaire à l'objet-frontière. Vers la prise en compte du travail d'équipement, Revue d'anthropologie des connaissances, 3, 1, 51-72. 